\title{
Research on Real-world Multidimensional Stroke Ontology and Its Reasoning for Construction of the Evolution Model
}

\author{
Luan Gao ${ }^{1,2}$, Fenggang $\mathrm{Li}^{3}$, Jian Wang ${ }^{1}$, Zhenguo Wang ${ }^{2}$, Lin $\mathrm{Luo}^{3}$ and Xiaozhi Gao ${ }^{4}$ \\ ${ }^{1}$ Anhui University of traditional Chinese medicine Hefei Anhui 230038 \\ ${ }^{2}$ Shandong University of traditional Chinese medicine Shandong Ji Nan 250355 \\ ${ }^{3}$ Hefei University of technology Hefei Anhui 230009 \\ ${ }^{4}$ Aalto University, Espoo, FI-00076, Finland
}

\begin{abstract}
The research planed to gather and standard the real world stroke clinical data from clinica to construct a stroke ontology corpus, through refactoring and sharing of data, established a multi-dimensional stroke ontology. And based on stroke diagnosis and treatment process, it integrated the evolution of reasoning mechanism research into the basic tier of theory, designed and implemented a multidimensional ontology the prototype experiment system which has building, reasoning, learning and evolution mechanism. The research results are expected to provide a shared system of concept of stroke to clinical diagnosis and treatment decisions, provide diagnosis and treatment strategy to optimize and design the stroke diagnosis and treatment plan, enhance the accuracy, objectivity and pertinency of diagnosis and treatment level, so as to provide the support of technical method for translating the application and development of medicine, which help to promote scientific, informatization, modernization of traditional Chinese medicine research.
\end{abstract}

Keywords-real world; multidimensional stroke ontology; reasoning; evolution; mentality

\section{INTRODUCTION}

Stroke has become one of the three major diseases threatening human health because of its characteristics of high incidence, high mortality and high morbidity, high recurrence rate and big quantity of complications. Therefore, the research on stroke has been the focus of the medical profession at home and abroad for a long time. It is a society full of information, the new situation of the research of traditional Chinese medicine which supported by "big data" is coming. Researches show that only in the real world, the advantages features of traditional Chinese medicine syndrome differentiation can be fully implemented and played. The use of clinical data in the process of the stroke clinical syndrome differentiation has important guiding significance for the correct decision of the clinical diagnosis and treatment. Therefore, carrying out a research into the real world stroke and sharing data of the clinical diagnosis and treatment, which have great theoretical value and practical significance.

As a kind of conceptual model, ontology can describe system on the semantic and knowledge level, which play important role in the field of Traditional Chinese medicine(TCM) of the knowledge mining, discovery and utilization. Based on the previous research, this topic will put the stroke as the breakthrough point, put forward a hypothesis on constructing a model of multidimensional stroke ontology and its reasoning evolution through a real world data. Follows are structure of this essay.

\section{THE RESEARCH STATUS AT HOME AND ABROAD}

A. The Real World Study of "Data Oriented" is the Only Way Which Must be Passed to the Current Clinical Research of Traditional Chinese Medicine

The real-world study(RWS)is a kind of new concept of clinical study. At present, it is an advanced concept which has been concerned by clinical research at home and abroad, particularly the clinical medical research[1]. The real world of clinical scientific research is mainly carried out by using clinical diagnosis and treatment records data. The various of information produced by daily clinical diagnosis and treatment practice in the real world can be preserved through a variety of forms such as medical records, physical and chemical detection, the doctor's advice records, hospitalization records and so on. In the process of treatment based on syndrome differentiation of traditional Chinese medicine produced a large number of principle-method-recipe-medicines information, the advantages of TCM syndrome differentiation and treatment can be fully implemented and expressed under the condition of the real world[2].

In recent years, the researchers use data information in data warehouse of hospital information system (HIS), based on the big data in the real world, carried out a series of research, the main goal is to study the rule of etiology and pathogenesis, syndrome characteristics, prescription drugs, etc[3]. The premise of develop the clinical research paradigm of the real world is to gathering and digital all kinds of clinical diagnosis and treatment information, and its innate character is a process of clinical practice facts quantitative[4].If the level of the Quantitative degree is high, then the level of datamation degree will be higher, which will do a favor to the information analysis and mining more specific in all kinds of inheritance contained in the clinical practice and innovation. Therefore, with the coming of the era of big data, "data oriented" in the real world research is the only way to the current clinical research of TCM.. 


\section{B. The TCM Ontology has a Good Application Prospect in the Research of Traditional Chinese Medicine}

Ontology is a concept originated from philosophy, it was a system description of objective existence in the world at the beginning, then it derivatived to the field of language, information, knowledge system, etc. At the moment, the definition of academic recognition is given by Gruber $\mathrm{T}$, that is ontology is clear specification explain of conceptual model[5].

Ontology is the formal specification for shared conceptual model and it has been widely used in various fields as a kind of modeling tool[6]. And how to extract and descript the knowledge in the different fields and construct the matched domain ontology, has become one of the hot research areas. The domain ontology is a kind of description of the subjects concept, ontology construction process are not identical to different subjects area and specific engineering discipline. Ontology construction method has always been a hot issue in the study of the ontology, at present, there are some comparative maturity method which can be used such as TOVE method, Methontolgy, skeleton method, KACTUS engineering method, SENSUS method, IDEF - five, seven steps, etc[7]. There is dozens of construction tools now and Ontoedit, Ontolingua, Webode, Wordmap, Protégé, KAON, Ontosaurus are common methods. The protégé is the most widely used tool in current domestic.

The researchers attach more and more importance to the construction of the medical domain ontology. Ontology construction of traditional Chinese medicine is based on the analysis of the related conceptions, result in expressing traditional Chinese medicine knowledge clearly and specifically, which will do good to knowledge's sharing and transfering. At present, ontology has got many achievements in traditional Chinese medicine application. For example, Lin Xiaohua etc. collected term in stroke rehabilitation from the ancient books of traditional Chinese medicine and then established ancient and modern glossary library of the stroke rehabilitation, which is helpful for communication between the ancient and modern concept in stroke rehabilitation field [8].Tian Tian etc. based on the theory documents of "lung and large intestine list", built the knowledge ontology of traditional Chinese medicine[9]. Li Jinghua built the ontology knowledge database of spleen and stomach disease of traditional Chinese of medicine for clinical decision support [10]. Cai Xiao-hong etc. has discussed the construction of traditional Chinese medicine ontology of senile dementia[11].Yu Tong etc. architecture semantic network framework of the language system of traditional Chinese medicine (TCM)[12].

As the important foundation of information processing of traditional Chinese medicine, ontology has a good application prospect in the TCM research. But now the TCM ontology building exist the following problems: (1)Lack of data from the real world. The existed TCM ontology are mostly come from ancient and modern literature and relevant data, thus lack of real world data from the clinical front line; (2)Lack of reasoning evolution mechanism research. With the development of the semantic Web, the reasoning mechanism study based on semantic interests researchers greatly. There is little research on specific domain ontology evolution, even the domain ontology of reasoning mechanism of traditional Chinese medicine evolution research is rarely reported. (3)Attaches great importance to the ontology construction but despise action mechanism research. Current researches are mostly based on the theoretical system of traditional Chinese medicine of ontology building, which often aim at how to build, but ignore the further study on its action mechanism.

\section{Ontology Reasoning Technology and Evolution Mechanism Research Become the Focus of the Current Domain Ontology Research}

For the ontology builder, ontology reasoning mechanism is mainly used to check the consistency of the ontology. For the ontology users, it can obtain knowledge and then apply it to solve the problem[13-14].With the development of semantic Web, ontology reasoning mechanism research based on semantic Web will become one of the hotspot in research of ontology[15-16].

At present, there are following four main method of realization of ontology reasoning[17]: 1)The method based on the traditional description logic reasoning. There are typical representative like Pellet, Racer and FaCT++. 2)Rule-based reasoning method. Such as Jess ontology inference machine system and Jena ontology reasoning machine system. 3)The method of using logic to program. Such as using the logic programming method of F-OWL ontology inference machine system and KAON2 of the university of karlsruhe in Germany. 4)The method based on first-order predicate prover, like Hoolet ontology reasoning machine, etc.

As we know, the knowledge is constantly updated, in order to make the ontology absorb and integrate the new intellectual, we need to alter the knowledge in ontology timely. Thus the ontology evolution become extremely important. Ontology evolution is also called the ontology development, it is a process and method which on the basis of existing domain ontology, according to the request of practical application, meanwhile, use a certain theory, methods and standards to enrich the ontology's structure, concept and the relationships. For a discipline domain ontology which possess strong applicability, the ontology evolution can be thought as the continuation of life ontology[18].

There are many studies about ontology evolution at home and abroad. Some scholars have put forward a kind of discovery-driven ontology evolution method[19]. Some proposed a ontology evolution patterns based on change operation framework. At the same time, some focused on the consistency validation of feature model evolution based on ontology, and some carried out the component-based software evolution information acquisition and measurement research based on ontology .

But now, most of the evolution mechanism research is universally applicable, there is a small amount of specific domain ontology evolution mechanism study, furthermore, the research on traditional Chinese medicine ontology evolution of reasoning mechanism are much less. So, the research on domain ontology reasoning evolution mechanism of traditional Chinese medicine has very important practical significance. 


\section{MODEL BUILDING RESEARCH}

The research for big data of the real world and integrate concept term which associated with stroke and finally establish a multi-dimensional stroke ontology. On the other hand, this study will blend in the reasoning evolution mechanism, designing and then realize the multidimensional stroke ontology and the prototype experiment system which have building, reasoning, learning and evolution mechanism.

\section{A. Collect Data from Real World Stroke Diagnosis and}

Treatment, and Turn It Normalization and Information

This study intends to select the information of diagnosis and treatment from Anhui province Chinese medicine hospital information management system (HIS) database and stroke patients clinical records, and standardization and information process with reference to 'National Standard of the People's Republic of China in Clinical diagnosis and treatment of traditional Chinese TCM terminology, syndrome part ', 'national standard of the People's Republic of China in the clinical diagnosis and treatment of TCM terminology, disease parts', 'National Standard of the People's Republic of China in Clinical diagnosis and treatment of traditional Chinese TCM terminology, treatment part',' Chinese Terms in TCM Pharmacy', 'Pharmacopoeia of the People's Republic' and so on.

\section{B. Build a Corpus of Stroke Ontology}

This study will be based on data in the real world, combined with domain terminology dictionary such as 'traditional Chinese medicine (TCM) keywords list', 'the Chinese medicine commonly used terms' etc. Take the related terminology as concepts of alternative in the ontology, build professional concept hierarchy, conduct the computer's structured processing and specification processing. In the end, using theory and technical methods of natural language processing (NLP) to rebuild the stroke concept system, using the hybrid ontology library strategy to construct a corpus of stroke ontology.

\section{Construct Multi-dimensional Stroke Ontology}

1) Ontology demand analysis. Ontology Demand is divided into functional requirements and non-functional requirements. For the purpose of this study, Functional requirements mainly describes the realization of this stroke diagnosis ontology, by contrast, the non-functional requirements mainly describes the performance indicators that need to be reached.

2) Ontology construction planning. This stage is mainly to do a overall planning for the target of research, the relevant of resources and the allocation of resources.

3) Complete ontology information acquisition. At this stage the main goal is to determine the information sources of knowledge ontology in TCM field, obtain the information data. Lay a foundation for the stroke diagnosis ontology construction.

4) Determine the ontology concepts and relationships. At this stage the main objective is to determine main concepts of the domain knowledge ontology, reveal various relationships between concepts, build the conceptual model of traditional Chinese medicine domain ontology.

5) Complete the ontology formalization encoding. At this stage the main goal is to use protégé tools and OWL language model as a build tool, describe ontology through the language of itself.

6) Ontology evaluation. At this stage the main goal is to assess and test the practicability of stroke diagnosis ontology established before.

7) Complete ontology expression. This stage mainly adopts the RDF technology to mark the structured TCM domain knowledge ontology ang displayed it clearly and orderly.

8) Build a multidimensional stroke ontology platform. The main target in this stage is to achieve the accuracy and systematic characterization of the stroke concept system, finally to provided a mesh stroke concept system with precise connotation and explicit extension for stroke research. Determine the top concept relations and its subclasses. At the same time, define class and class hierarchy, define the attributes of class and split faceted of custom properties.

\section{For ontology Library Construction Strategy Based on Medical Cases, Construct an Stroke Diagnosis and Treatment Ontology}

First of all, the project introduce TCM ontology into object putted forward model, enhance semantic expression of putted forward model, simplify the resolving process of semantic conflict, combined the ontology method with object-oriented database technology, apply into the establishment of the stroke putted forward model. The medical ontology library model consists three layers structure, from top to bottom respectively are the application layer, semantic layer and data layer.

Secondly, adopt the strategy of hybrid ontology library to build ontology library of stroke putted forward model, which include global ontology library and local ontology library. Global ontology library, that is the concept dictionary which formed in the process of stroke diagnosis ontology construction, which is built by the integration of the medical language system and the method of ontology.

Finally, on the basis of the concept dictionary, using method of ontology study, through the loop learning to concept dictionary with several stages like importing, reusing, extracting, pruning, refining and application of assessment, local ontology library of stroke case model can be built by choosing sub-ontology from a large ontology. Specific description are as follows:

\section{E. Study on Medical Ontology Reasoning Learning and Evolution Mechanism Face in the field of Traditional Chinese Medicine, Provide the Basis For the Evolution of Stroke Diagnosis Ontology Reasoning}

Firstly, study on the medical ontology integration mechanism, including ontology mapping, ontology binding and the ontology fusion. Secondly, study on medical ontology reasoning learning mechanism face in the field of traditional 
Chinese medicine, discuss the existing ontology reasoning machine through comparing, look for suitable reasoning mechanism for stroke diagnosis and treatment ontology. Finally, study on medical ontology reasoning evolution mechanism face in the field of traditional Chinese medicine. On the one hand, make the fully understanding of the existing working principle of the evolution mechanism, to explore evolution mechanism which suitable for the stroke diagnosis ontology. On the other hand, try to fuse the case-based reasoning technology into the stroke ontology evolution mechanism.

\section{F. Based on Stroke Diagnosis and Treatment Process, Design} and Implement Prototype Experiment System with

Ontology Construction, Reasoning, Learning And Evolution Mechanism

In order to validate the theory and method put forward in this project, meanwhile for the experiment platform can be used in future research, we constructed a stroke diagnosis ontology reasoning prototype experiment system. As shown in the following figure:

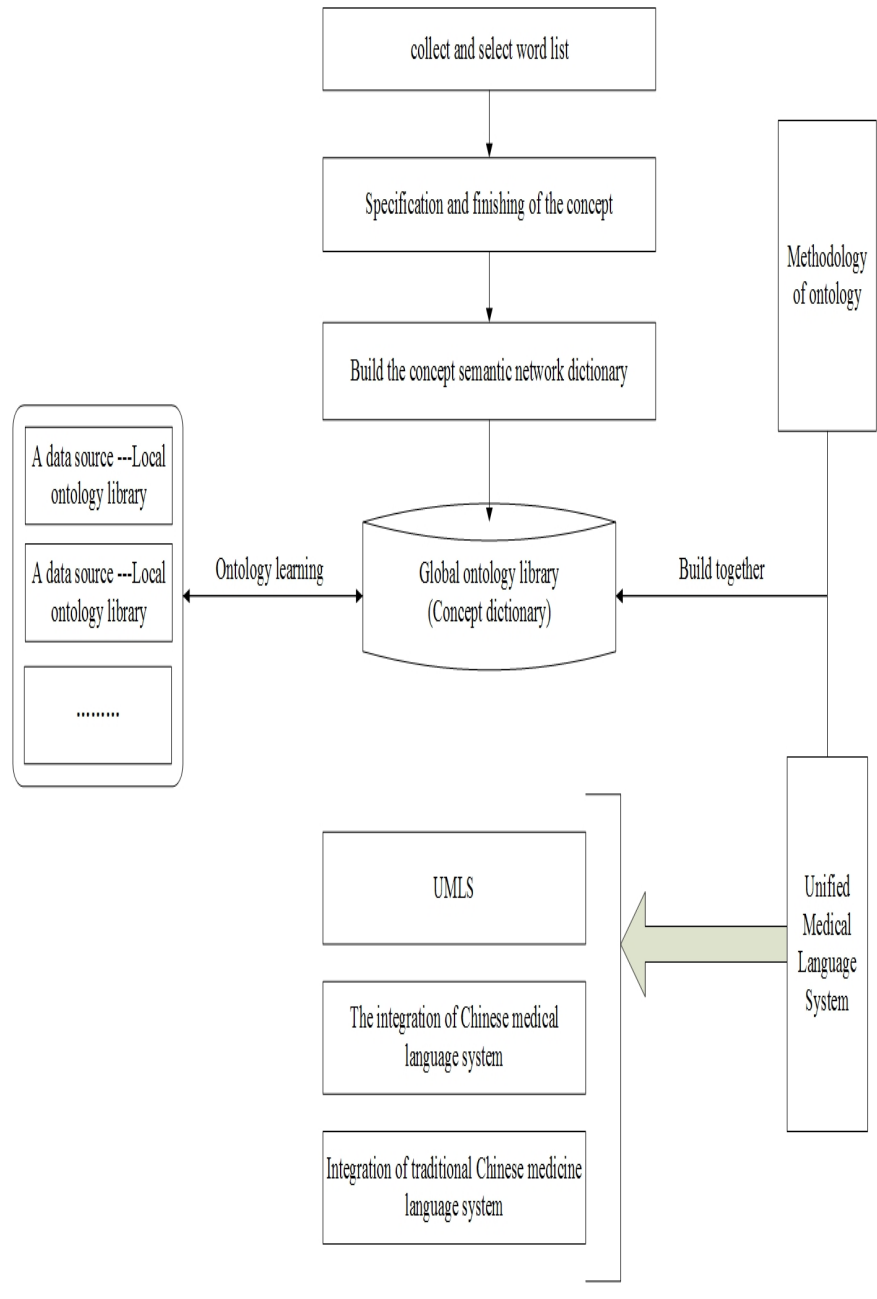

FIGURE I. THE ONTOLOGY TECHNOLOGY ROADMAP BASED ON HYBRID ONTOLOGY LIBRARY CONSTRUCTION OF STRATEGY OF STROKE CASE LIBRARY MODEL

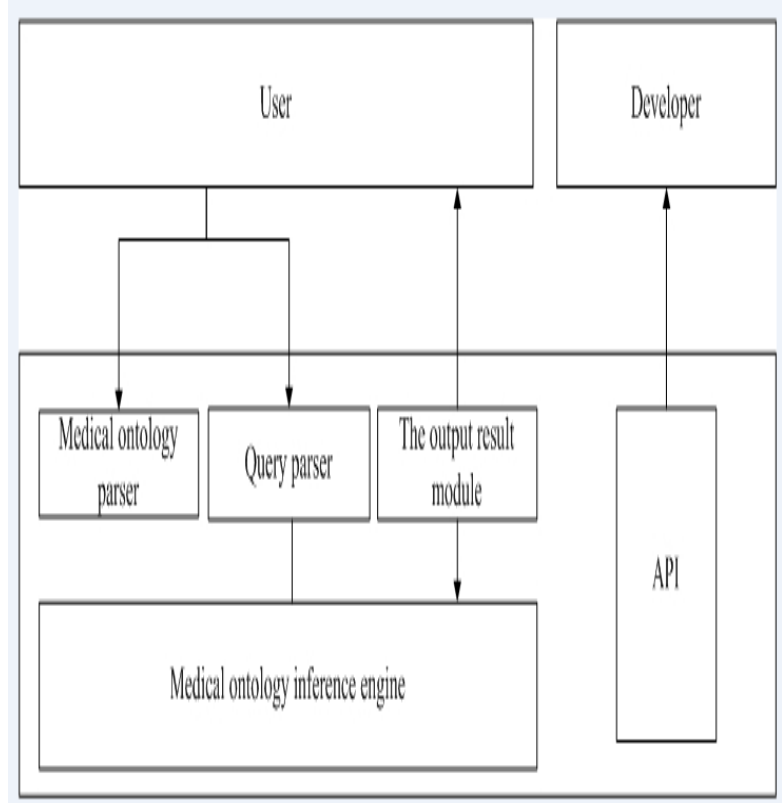

FIGURE II. MEDICAL ONTOLOGY REASONING PROTOTYPE EXPERIMENT SYSTEM STRUCTURE CHART

\section{The Overall Technical Route}

As shown in the following figure :

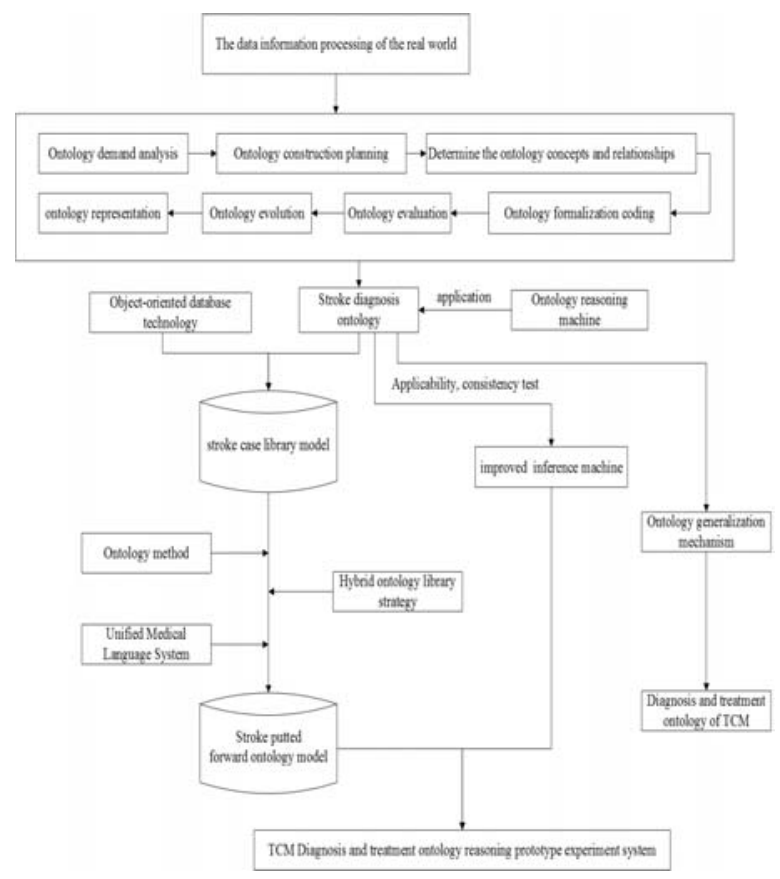

FIGURE III. THE TOTAL TECHNOLOGY ROADMAP

According to the big data of the real world, This study will use the theory and technical method of natural language processing (NLP) to rebuild the concept of the term that associated with stroke diagnosis and treatment, then to establish a multidimensional stroke ontology, finally realize the refactoring and sharing of knowledge. In the meantime, this 
research will blend the evolution of reasoning mechanism in the basic theory to study, design and bring about a prototype experiment system and multidimensional stroke ontology which have the function of building, reasoning, learning and evolution mechanism.

\section{CONCLUSION}

The clinical scientific research in the real world has very important significance for the traditional Chinese medicine and the information of the data is the most important part. This topic put the stroke as the breakthrough point, come up with a hypothesis that about constructing model of multidimensional stroke ontology and its reasoning evolution through a real world data. The research results are expected to provide a shared system of concept of stroke to clinical diagnosis and treatment decisions, provide diagnosis and treatment strategy to optimize and design the stroke diagnosis and treatment plan, enhance the accuracy, objectivity and pertinency of diagnosis and treatment level, so as to provide the support of technical method for translating the application and development of medicine, which help to promote scientific, information, modernization of traditional Chinese medicine research. What's more, this essay will also provide a new research thought for the construction of ontology evolution mechanism in other field.

\section{ACKNOWLEDGMENT}

This research has been supported by the National Natural Science Fund under Grant No. 81102547 and Anhui Province research fund for the returned overseas talent scholars(2015). We sincerely thank these funds support.

\section{REFERENCES}

[1] Runshun Zhang, Yinghui Wang, Baoyan Liu, Guangli Song, Xuezhong Zhou, Shizhen Fan, Xishui Pan. Clinical data quality problems and countermeasure for real world study[J]. Frontiers of Medicine, 2014,8(3):352-357.

[2] Dong X,Cao K, Ma B,Gao Y.briefly research on traditional Chinese medicine terminology of the real world at the era of big data[J] .China Journal of Traditional Chinese Medicine and Pharmacy,2014,29(9):2724-2726.

[3] Jiefeng C,Wei Y, Yanming X,Yong S ,Yan Z,Yongyan W.Characteristics and drug analysis associated with vertigo disease in real world[J].Chinese journal of traditional Chinese medicine,2014,39(18):3514-3518.

[4] GruberT. A translation approach to portable ontology specifications[J]. Knowledge Acquisition, 1993,5(2):199-220

[5] Duy Dinh.Identifying change patterns of concept attributes in ontology evolution[M].The Semantic Web: Trends and Challenges Lecture Notes in Computer Science Volume 8465, 2014.768-783.

[6] Yuan Y.A Study on Methodologies and Tools for Building Domain Ontologies[M]. Beijing:China renmin university press, 2004. system,2015,24(1):65-68.

[7] Wang Y,Wang L,Jiang S,Huang Y.Medical terminology oriented ontology model and its service system design[J].Computer Engineering and Design,2015,36(1):258-262.

[8] Li J, Yi X. Research for Clinical Decision Support of Spleen and Stomach Disease of TCM Ontology Knowledge Base Building[J].Chinese medical innovation,2014,11(27):120-125.

[9] Tian T,Gao S,Zhao D ,Mo Fangfang, Ma shuai lei,Ma yue, et al.TCM knowledge ontology construction based on the theory research of 'lung and large intestine being interior-exteriorly related'[J].Journal of Chinese medicine,2013(28)5;1183-1188.

[10] Cai X,Kong M,Wang P. Study on Senile dementia of traditional Chinese medicine ontology construction [J].When Jane national physician characters,2014,25(8):1918-1919.

[11] Lin X,Lin D,Tao J.Method of Constructing Ancient and Terminology Data based of Stroke Rehabilitation Based on Ontology[J].Chinese journal of Chinese medicine information, 2012,19(1):32-35.

[12] Yu T,Cui M,Li H.Semantic Network Framework of Traditional Chinese Medicine Language System:An Upper-Level Ontology for Traditional Chinese Medicine[J].China Digital Medicine ,2010,19(9):163-167.

[13] Pan C,Gu H.Ontology Reasoner and Its Application. The computer system application,2014,9(1):43-47.

[14] Hammar, K. Reasoning Performance Indicators for Ontology Design Patterns[J].CEUR Workshop Proceedings,2014.2-13.

[15] Yuwei Zhang, Zhiqiang Wei, Yongquan Yang. Ontology Description of Smart Home Appliance Based on Semantic Web[J].International Conference on Computer Science and Service System.2012.695-698.

[16] Sirin E. Pellet:A practical OWL-DL reasoner. Journal of Web Semantics,2006.

[17] C. Maria Keet .The Data Mining OPtimization Ontology[J].Web Semantics: Science, Services and Agents on the World Wide Web,2015.1(001):1-11.

[18] Castano S,Ferrara A,Hess NG. Discovery-driven ontology evolution.Pisa:Scuol a Normale Superiore,2006

[19] Javed M, Yalemisew M, Abgaz, Pahl C.A pattern-based framework of change opera tors for ontology evolution[J].In:Shvaiko P,eds. The 4th International Workshop on Ontology Content. Algarve: OTM Workshops,2009.544-553 